\title{
Perfect Dominating Sets in Fuzzy Graphs
}

\author{
S. Revathi, P.J.Jayalakshmi, C.V.R.Harinarayanan \\ Assistant Professor,Department of Mathematics,Saranathan College of Engineering, Trichy, Tamilmadu, India \\ †Associate Professor,Department of Mathematics, Indra Ganesan college of Engineering, Trichy, Tamilnadu, India \\ $\ddagger$ Research Supervisor\&Assistant Professor, Department of Mathematics, Government Arts College, Paramakudi, \\ Tamilnadu,India
}

\begin{abstract}
The basic definitions of fuzzy independent set, fuzzy dominating set and fuzzy independent dominating sets are discussed. In this paper we introduce the concept of perfect domination in fuzzy graphs and obtain some interesting results for this new parameter in fuzzy graphs and the aim of this paper is to find on what relations the fuzzy graph has perfect domination number and independent domination number. Finally, the independent domination number for a connected fuzzy graph is obtained.
\end{abstract}

Key words: Fuzzy graph,Fuzzy dominating set, fuzzy independent domi-nating set, perfect dominating set .

\section{Introduction}

Harary et al. [1] explained an interesting application in voting situations using the concept of domination. The study of dominating sets in graphs was begun by Ore and Berge, the domination number, independent domi- nation number are introduced by Cockayne and Hedetniemi. Rosenfeld [11] introduced the notion of fuzzy graph and several fuzzy analogs of graph the- oretic concepts such as paths, cycles and connectedness. A.Somasundram and S.Somasundram [13] discussed domination in fuzzy graph.They defined domination using effective edges in fuzzy graph. Nagoor Gani and Chandrasekaran [8] discussed domination in fuzzy graph using strong arcs. We also discuss domination, independent domination and perfect domination in fuzzy graph using strong arcs. Nagoorgani and Vadivel [9] discussed fuzzy independent dominating sets. In this paper we discuss when the fuzzy graph has perfect domination and independent domination number. The necessary definitions are given and explained with examples. Some fuzzy graphs also compared with the crisp case.

\section{Preliminaries}

A fuzzy subset of a nonempty set $\mathrm{V}$ is a mapping $\sigma: \mathrm{V} \rightarrow[0,1]$. A fuzzy relation on $\mathrm{V}$ is a fuzzy subset of $V x V$. A fuzzy graph $G=(\sigma, \mu)$ is a pair

of function $\sigma: \mathrm{V} \rightarrow[0,1]$ and $\mu: \mathrm{V} x \mathrm{~V} \rightarrow[0,1]$, where $\mu(\mathrm{u}, \mathrm{v}) \leq \sigma(\mathrm{u}) \mathrm{V} \sigma(\mathrm{v})$

for all $u, v$ V. The underlying crisp graph of $G=(\sigma, \mu)$ is denoted by $G^{*}=(V, E)$, where $V=\{u \quad V$ : $\sigma(\mathrm{u})>0\}$ and $\mathrm{E}=\{(\mathrm{u}, \mathrm{v}) \quad \mathrm{VxV}: \mu(\mathrm{u}, \mathrm{v})>0\}$. The order

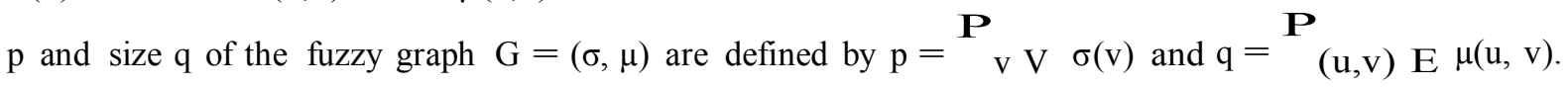
$\mathbf{P}$

Further let $\mathrm{G}$ be a fuzzy graph on $\mathrm{V}$ and $\mathrm{S} \subseteq \mathrm{V}$, then the fuzzy cardinality of $\mathrm{S}$ is defined to be $\mathrm{v} \mathrm{S} \sigma(\mathrm{v})$. The strength of the connect- edness between two nodes $u, v$ in a fuzzy graph $G$ is $\mu^{\infty}(u, v)=\sup \left\{\mu^{k}(u, v)\right.$

$\mathrm{k}=1,2,3 \cdots$,$\} , Where \mu^{\mathrm{k}}(\mathrm{u}, \mathrm{v})=\sup \left\{\mu(\mathrm{u}, \mathrm{u} 1){ }^{\mathrm{V}} \mu\left(\mathrm{u}_{1}, \mathrm{u}_{2}\right){ }^{\mathrm{V}}{ }^{\mathrm{V}}{ }_{\left.\mu\left(\mathrm{uk}_{\mathrm{k}}-1, \mathrm{v}\right)\right\}}\right.$.

An $\operatorname{arc}(u, v)$ is said to be a strong arc or strong edge, if $\mu(u, v) \geq \mu^{\infty}(u, v)$ and the nodev is said to be strong neighbor of $u$. A node $u$ is said to be isolated if $\mu(\mathrm{u}, \mathrm{v})=0$ for all $\mathrm{u}=\mathrm{v}$. In a fuzzy graph, every arc is a strong arc then the graph is called strong arc fuzzy graph. Let $\mathrm{u}$ be a node in fuzzy graph $\mathrm{G}$ then $\mathrm{N}(\mathrm{u})=\{\mathrm{v}:(\mathrm{u}, \mathrm{v})$ is a strong $\operatorname{arc}\}$ is called neighborhood of

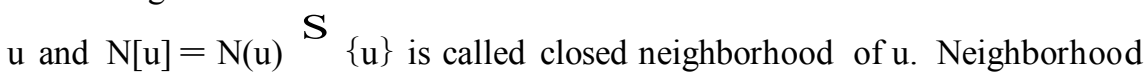

degree of the node is defined by the sum of the weights of the strong neigh- bor node of $u$ and is denoted 
by $\mathrm{d}_{\mathrm{N}}(\mathrm{u})={ }_{\mathrm{v} V}^{\mathbf{P}} \sigma(\mathrm{v})$. Minimum neighborhood

degree of a fuzzy graph $\mathrm{G}$ is defined by $\delta_{\mathrm{N}}(\mathrm{G})=\left\{\operatorname{mind}_{\mathrm{N}}(\mathrm{u}): \mathrm{u} \mathrm{V}(\mathrm{G})\right\}$ and maximum neighborhood degree of $\mathrm{G}$ is by $\Delta \mathrm{N}(\mathrm{G})=\left\{\operatorname{maxd}_{\mathrm{N}}(\mathrm{u}): \mathrm{u} \mathrm{V}(\mathrm{G})\right\}$

\section{Fuzzy Dominating Set}

Definition 2.1 Let $G=(\sigma, \mu)$ be a fuzzy graph. A subset $\mathrm{D}$ of $\mathrm{V}$ is said to be a dominating set of $\mathrm{G}$ if for every $\mathrm{v} \mathrm{V}$ - $\mathrm{D}$, there exists a $\mathrm{u} \mathrm{D}$ such that $\mathrm{u}$ dominates $\mathrm{v}$.

Definition 2.2 A dominating set D of a fuzzy graph $G$ is called minimal dominating set of $G$ if there does not exist any dominating set of G, whose cardinality is less than the cardinality of D. Minimum cardinality among all minimum dominating set in $\mathrm{G}$ is called domination number of $\mathrm{G}$ is denoted by $\gamma(\mathrm{G})$.

\section{Fuzzy Independent Set}

Definition 3.1 Let $G=(\sigma, \mu)$ be a fuzzy graph. Two nodes in a fuzzy graph $G$ are said to be fuzzy independent if there is no strong arc between them. A subset $\mathrm{S}$ of $\mathrm{V}$ is said to be fuzzy independent set for $\mathrm{G}$ if every two nodes of $\mathrm{S}$ are fuzzy independent.

Definition 3.2 Let $G=(\sigma, \mu)$ be a fuzzy graph. A fuzzy independent set $S$ of $G$ is said to be maximal fuzzy independent set if there is no fuzzy independent set whose cardinality is greater than the cardinality of S. The maximum car- dinality among all maximal fuzzy independent set is called fuzzy independence number of $\mathrm{G}$ and it is denoted by $\beta(\mathrm{G})$.

\section{Perfect Dominating Set}

Definition 4.1 A dominating set $\mathrm{D}$ of a fuzzy graph $\mathrm{G}$ is said to be a perfect dominating set if for each vertex $v$ not in $D$, $v$ is adjacent to exactly one vertex of $D$.

Definition 4.2 A perfect dominating set $\mathrm{D}$ of a fuzzy graph $\mathrm{G}$ is said to be a minimal perfect dominating set if for each vertex $\mathrm{v}$ in $\mathrm{D}, \mathrm{D}-\{\mathrm{v}\}$ is not a dominating set. A perfect dominating set with smallest cardinality is called minimum perfect dominating set. It is denoted by $\gamma_{\mathrm{pf}}$ set of G. The cardi- nality of a minimum perfect dominating set is called the perfect domination number of the fuzzy graph G. It is denoted by $\gamma_{\mathrm{pf}}(\mathrm{G})$.

Definition 4.3 Let $G=(\sigma, \mu)$ be a fuzzy graph such that its crisp graph is a cycle, then $G$ is called a fuzzy cycle if there does not exists a unique arc $(\mathrm{x}$,

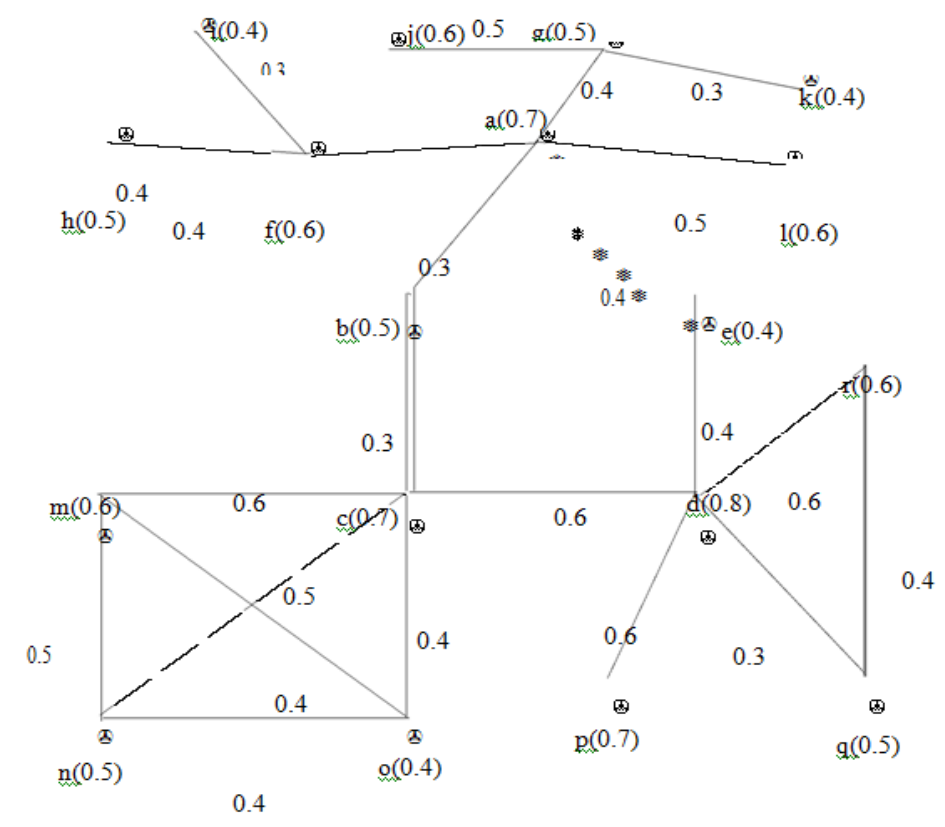


here $\mathrm{D}=\{\mathrm{a}, \mathrm{f}, \mathrm{g}, \mathrm{p}, \mathrm{r}, \mathrm{o}\} \mathrm{D}^{\prime}=\{\mathrm{b}, \mathrm{c}, \mathrm{d}, \mathrm{e}, \mathrm{h}, \mathrm{i}, \mathrm{j}, \mathrm{k}, \mathrm{l}, \mathrm{m}, \mathrm{n}, \mathrm{q}\}$

Theorem 4.4 Every complete fuzzy graph $\mathrm{G}$ is a perfect dominating set.

Proof:- G is a complete fuzzy graph. Therefore every arc in G is strong arc (ie) every vertex v V-D is adjacent to exactly one vertex of the dominating set D in G. Hence D is a perfect dominating set. Therefore, every complete fuzzy graph $\mathrm{G}$ is a perfect dominating set.

Theorem 4.5 A perfect dominating set $\mathrm{D}$ of a fuzzy graph $\mathrm{G}$ is minimal perfect dominating set if and only if for each vertex $\mathrm{v}$ in $\mathrm{D}, \mathrm{P}_{\mathrm{nf}}[\mathrm{v}, \mathrm{D}]$ is non-empty.

Proof:- Suppose D is minimal and $\mathrm{v} D$. Therefore there is a vertex $w$ not in D- $\{\mathrm{v}\}$ such that $w$ is adjacent to no vertex of $\mathrm{D}-\{\mathrm{v}\}$ or $\mathrm{w}$ is adjacent to atleast two vertices of $\mathrm{D}-\{\mathrm{v}\}$. If $\mathrm{w}=\mathrm{v}$ then this implies that $v P_{n f}[v, D]$. If $w=v$ then it is impossible that $w$ is adjacent to atleast two vertices of D$\{v\}$ because $D$ is a perfect dominating set. Therefore $w$ is not adjacent to any vertex of $D-\{v\}$. Since $\mathrm{D}$ is a perfect dominating set. $\mathrm{W}$ is adjacent to only

$\mathrm{v}$ in $\mathrm{D}(\mathrm{ie}) \mathrm{N}(\mathrm{w}){ }^{\mathrm{T}} \mathrm{D}=\mathrm{v}$. Thus, $\mathrm{w} \mathrm{P}_{\mathrm{nf}}[\mathrm{v}, \mathrm{D}]$. Conversely, suppose $\mathrm{v} \mathrm{D}$ and

$P_{n f}[v, D]$ contains some vertex $w$ of $G$. If $w=v$ then $w$ is either adjacent to atleast two vertices of D-

$\{\mathrm{v}\}$ or $\mathrm{w}$ is adjacent to no vertex of $\mathrm{D}-\{\mathrm{v}\}$. Thus $\mathrm{D}-\{\mathrm{v}\}$ is not a perfect dominating set. If $\mathrm{w}=\mathrm{v}$ then $\mathrm{N}(\mathrm{w})^{\mathrm{T}} \mathrm{D}=\mathrm{v}$ implies that

$\mathrm{w}$ is not adjacent to any vertex of $\mathrm{D}-\{\mathrm{v}\}$. Thus, in all cases $\mathrm{D}-\{\mathrm{v}\}$ is not a perfect dominating set if $\mathrm{v}$ D. Thus, D is minimal.

Theorem 4.6 Let $\mathrm{D}_{1}$ and $\mathrm{D}_{2}$ be two disjoint perfect dominating sets of a fuzzy graph G. Then $\left|\mathrm{D}_{1}\right|$ $=\left|D_{2}\right|$

Proof:- For every vertex $\mathrm{x}$ in $\mathrm{D}_{1}$ there is a unique vertex $\mathrm{v}(\mathrm{x})$ in $\mathrm{D}_{2}$ which is adjacent to $\mathrm{x}$. Also, for every vertex y in $\mathrm{D}_{2}$ there is a unique vertex $\mathrm{u}(\mathrm{y})$ in $\mathrm{D}_{1}$ which is adjacent to $\mathrm{y}$. It may be noted that these functions are inverses of each other. Therefore, $\left|\mathrm{D}_{1}\right|=\left|\mathrm{D}_{2}\right|$

Corrolary 4.7 If in a fuzzy graph $G$ there are perfect dominating sets $D_{1}$

and $\mathrm{D}_{2}$ such that $\left|\mathrm{D}_{1}\right|=\left|\mathrm{D}_{2}\right|$ then $\mathrm{D}_{1}{ }^{\mathrm{T}} \mathrm{D}_{2}=\varphi$

Corrolary 4.8 Let $\mathrm{G}$ be a fuzzy graph with $\mathrm{n}$ vertices. If there is a per- fect dominating set $\mathrm{D}$ with $|\mathrm{D}|<\mathrm{n} / 2$ or $\geq \mathrm{n} / 2$ then $\mathrm{V}-\mathrm{D}$ is not a perfect dominating set.

Theorem 4.9 A perfect dominating set D of a fuzzy graph $\mathrm{G}$ is a minimal dominating set if and only if for each $\mathrm{v} \mathrm{D}$ one of the following two conditions holds.

(i) $\mathrm{N}(\mathrm{v})^{\mathrm{T}} \mathrm{D}=\varphi$

(ii) There is a vertex w V-D such that $\mathrm{N}(\mathrm{w}){ }^{\mathrm{T}} \mathrm{D}=\{\mathrm{v}\}$

Proof:- Let $\mathrm{D}$ be a minimal perfect dominating set and $\mathrm{v} \mathrm{D}$. Then $\mathrm{D}^{\prime}=\mathrm{D}-$

$\{\mathrm{v}\}$ is not a dominating set and hence there exists $\mathrm{x}$ V-D' such that $\mathrm{x}$ is not dominated by an element of D'. If $x=v$ we get (i) and if $x=v$ we get (ii). The converse is obvious. Theorem 4.10 Let $G$ be a connected fuzzy graph and let $\mathrm{D}$ be a minimal perfect dominating set of G. Then V-D is a dominating set of $\mathrm{G}$.

Proof:- Let D be a minimal perfect dominating set of G. suppose V-D is not a dominating set. Then there exists a vertex $v \mathrm{D}$ such that $\mathrm{v}$ is not a dominated by any vertex in $\mathrm{V}-\mathrm{D}$. since $\mathrm{G}$ is connected, $\mathrm{v}$ is a strong neighbor of atleast one vertex in $\mathrm{D}-\{\mathrm{v}\}$. Then $\mathrm{D}-\{\mathrm{v}\}$ is a dominating set, which contradicts the minimality of $\mathrm{D}$. Thus every vertex in $\mathrm{D}$ is a strong neighbor of atleast one vertex in V-D. Hence V-D is a dominating set.

Theorem 4.11 Every minimum perfect dominating set of a fuzzy graph G 
is not a minimal perfect dominating set.

The converse of this result need not be true.

Proposition 4.12 Let $G=(\sigma, \mu)$ be a fuzzy graph. Let $\mathrm{D}$ be a perfect dom- inating set with the perfect domination number $\gamma_{\mathrm{pf}}(\mathrm{G})$ and $\mathrm{i}(\mathrm{G})$ denotes the independent domination number. Then $\gamma_{\mathrm{pf}}(\mathrm{G}) \leq$ $\mathrm{i}(\mathrm{G})$.

Theorem 4.13 Let $G=(\sigma, \mu)$ be a fuzzy graph. Let $D$ be a minimum perfect dominating set with the perfect domination number $\gamma_{\mathrm{pf}}(\mathrm{G})$. The subgraph $\mathrm{hDi}$ induced by $\mathrm{D}$ has isolated nodes (i.e) $\mu(\mathrm{u}, \mathrm{v})=0$ for all $\mathrm{u}, \mathrm{v} \quad \mathrm{D}$ then $\gamma_{\mathrm{pf}}(\mathrm{G})$

$=\mathrm{i}(\mathrm{G})$ where $\mathrm{i}(\mathrm{G})$ denotes the independent domination number.

Proof:- It is clear from the definition that the minimum perfect dominating set $\mathrm{D}$ is the smallest perfect dominating set among all minimal perfect dom- inating sets. Since the subgraph induced with the nodes of $D$ are isolated implies that they are independent. Hence $\gamma_{p f}(G)=i(G)$. In comparing to the crisp case, $\gamma_{\mathrm{pf}}(\mathrm{G})=\mathrm{i}(\mathrm{G})$ if the graph $\mathrm{G}$ is claw free but that is not required for fuzzy graph.

Corrolary 4.14 Let $\mathrm{G}=(\sigma, \mu)$ be a fuzzy line graph. If the subgraph induced by $\mathrm{D}$ has isolated nodes then $\gamma_{\mathrm{pf}}(\mathrm{L}(\mathrm{G}))=\mathrm{i}(\mathrm{L}(\mathrm{G}))$.

Corrolary 4.15 If $\mathrm{G}=(\sigma, \mu)$ is a complete fuzzygraph then $\mathrm{i}(\mathrm{G})<\gamma_{\mathrm{pf}}(\mathrm{G})$.

Proof:- Since $G$ is a complete fuzzy graph every arc in $G$ is a strong arc. Hence $i(G)=0$ and $\gamma(G)=$ $\mathrm{V}\{\sigma(\mathrm{v})$; for all $\mathrm{v} \mathrm{V}\}$ and $\mathrm{i}(\mathrm{G})=0$. It is clear

that $\mathrm{i}(\mathrm{G})<\gamma_{\mathrm{pf}}(\mathrm{G})$.

Definition 4.16 A fuzzy graph $G=(\sigma, \mu)$ is said to be connected if there exists a strongest path between any two nodes of $\mathrm{G}$.

Definition 4.17 Let $\mathrm{u}$ be a node in fuzzy graph $\mathrm{G}$ then $\mathrm{N}(\mathrm{u})=\{\mathrm{v}:(\mathrm{u}, \mathrm{v})$ is a strong arc $\}$ is called neighborhood of $\mathrm{u}$ and $\mathrm{N}[\mathrm{u}]=\mathrm{N}(\mathrm{u}) \mathrm{S} u$ is called closed

neighborhood of $u$. Neighborhood degree of the node is defined by the sum of the weights of the strong neighbor node of $\mathrm{u}$ and is denoted by $\mathrm{d} \mathrm{N}(\mathrm{u})=$

$\mathbf{P}$

v N(u) $\sigma(v)$

Theorem 4.18 Let $\mathrm{G}=(\sigma, \mu)$ be connected fuzzy graph which does not have an induced fuzzy cycle subgraph. Let $\mathrm{D}$ be a minimum perfect domi- nating set with perfect domination number $\gamma_{\mathrm{pf}}(\mathrm{G})$. Then $\mathrm{i}(\mathrm{G})=\beta(\mathrm{Y})+$

$\mathbf{P}$

$u(D-Y) d(N(u))$ where $Y$ is maximal independent set of $D$.

Proof:- The nodes in the perfect dominating set $\mathrm{D}$ is also connected since the fuzzy graph $\mathrm{G}$ is connected. It shows that $\gamma_{\mathrm{pf}}(\mathrm{G})=\mathrm{i}(\mathrm{G})$. Let $\mathrm{Y}$ denote the maximal independent set in $\mathrm{D}$ and its cardinality is $\beta(Y)$. The nodes in V-D are independent if not they would have an induced fuzzy cycle which contradicts our assumption. Since the nodes in D-Y are not independent its corresponding neighbors are independent. Hence $\mathrm{i}(\mathrm{G})=$

$\beta(\mathrm{Y})+{ }^{\mathbf{P}} \mathrm{u}(\mathrm{D}-\mathrm{Y}) \mathrm{d}(\mathrm{N}(\mathrm{u}))$

\section{References}

[1]. Auer, D.B., Harary, F., Nieminen, J., and C.L. Suffel. Domination Alteration Sets in Graphs,Discrete Math., 47:153$161,1983$.

[2]. Bollobas, B., and Cockayne, E.J., Graph theoretic parameters con-cerning domination,independence and irredundance. J. Graph Theory, 3:241-250,1979.

[3]. Brigham, C., Chinn, Z., and Dutton, D., Vertex Domination - Critical Graphs, Networks, Vol. 18 (1988) 173-179.

[4]. Carrington, J.R., Harary, F., and Haynes, T.W., Changing and un- changing the domination number of a graph. J.Combin., Math. Com- bin. Comput., 9: 57-63, 1991. 
[5]. Sumner, D.P., and Blitch, P., Domination critical graphs ,J.Combin, Theory Ser. B, 34:65-76, 1983.

[6]. Haynes, T., Hedetniemi,S.T., Slater, P.J., Fundamentals of domination in graph, Marcel Deckker, New York, 1998.

[7]. Nagoorgani, A., Vijayalakshmi, P.., Fuzzy Graphs With Equal Fuzzy Domination And Independent Domination Numbers, International Jour- nal of Engineering Science and Technology Development, Vol.1,No.2 (2012)66-68.

[8]. Nagoorgani,A., and Chandrasekaran,V.T., Domination in fuzzy graph, Advances in fuzzy sets and system I(1)(2006), 17-26.

[9]. Nagoorgani, A., and Vadivel, P., Fuzzy independent dominating set, Adv. in Fuzzy sets and system 2(1) (2007), 99-108.

[10]. Nagoorgani,A., Vadivel,P., Relations between the parameters of Inde- pendent Domination and Irredundancein Fuzzy Graph, International Journal of Algorithms, Computing and Mathematics, Volume 2, Num- ber 1, pp. 15-19, 2009.

[11]. Rosenfeld, A., Fuzzy graphs in: Zadeh, L.A., Fu, K.S., Shimura, M (eds)., Fuzzy Sets and Their Applications, Academic Press, New York,1975.

[12]. Nagoorgani, A., Vijayalakshmi, P., Domination Critical Nodes in Fuzzy Graph. International J. of Math. Sci. \& Engg. Appls. (IJMSEA), Vol.5. No. I , pp.295-301(2011).

[13]. Somasundaram, A., and Somasundaram, S., Domination in fuzzy graphs, Pattern Recognit. Lett. 19(9) 1998), 787-791.

[14]. Teresa W. Haynes, Stephen T. Hedetniemi and Peter J. Slater, Funda- mentals of Domination in Graphs, Marcel Dekker, Inc., New York. 